Research

Open Access

\title{
Gastrointestinal Failure score in critically ill patients: a prospective observational study
}

\author{
Annika Reintam ${ }^{1,2}$, Pille Parm ${ }^{3}$, Reet Kitus ${ }^{1,3}$, Joel Starkopf ${ }^{1,3}$ and Hartmut Kern ${ }^{1,4}$
}

${ }^{1}$ Clinic of Anaesthesiology and Intensive Care, University of Tartu, Puusepa, Tartu 51014, Estonia

2Department of Anaesthesiology and Intensive Care, East Tallinn Central Hospital, Ravi, Tallinn 10138, Estonia

${ }^{3}$ Clinic of Anaesthesiology and Intensive Care, Tartu University Hospital, Puusepa, Tartu 51014, Estonia

${ }^{4}$ Klinik für Anästhesiologie und Intensivmedizin, DRK Kliniken Berlin Köpenick, Salvador-Allende-Straße, Berlin 12559, Germany

Corresponding author: Annika Reintam, annika.reintam@itk.ee

Received: 18 Mar 2008 Revisions requested: 22 Apr 2008 Revisions received: 8 Jul 2008 Accepted: 14 Jul 2008 Published: 14 Jul 2008

Critical Care 2008, 12:R90 (doi:10.1186/cc6958)

This article is online at: http://ccforum.com/content/12/4/R90

(C) 2008 Reintam et al.; licensee BioMed Central Ltd.

This is an open access article distributed under the terms of the Creative Commons Attribution License (http://creativecommons.org/licenses/by/2.0), which permits unrestricted use, distribution, and reproduction in any medium, provided the original work is properly cited.

\begin{abstract}
Introduction There are no universally accepted diagnostic criteria for gastrointestinal failure in critically ill patients. In the present study we tested whether the occurrence of food intolerance (FI) and intra-abdominal hypertension (IAH), combined in a 5-grade scoring system for assessment of gastrointestinal function (the Gastrointestinal Failure [GIF] score), predicts mortality. The prognostic value of the GIF score alone and in combination with the Sequential Organ Failure Assessment (SOFA) score is evaluated, and the incidence and outcome of gastrointestinal failure is described relative to the GIF score.
\end{abstract}

Methods A total of 264 subsequently hospitalized patients, who were mechanically ventilated on admission and stayed in the intensive care unit (ICU) for longer than 24 hours, were prospectively studied. GIF score was documented daily as follows: $0=$ normal gastrointestinal function; $1=$ enteral feeding with under $50 \%$ of calculated needs or no feeding 3 days after abdominal surgery; $2=\mathrm{FI}$ or $\mathrm{IAH} ; 3=\mathrm{FI}$ and $\mathrm{IAH}$; and $4=$ abdominal compartment syndrome (ACS). Admission parameters and mean GIF and SOFA scores for the first 3 days were used to predict ICU outcome.

Results $\mathrm{FI}$ developed in 58.3\%, IAH in $27.3 \%$, and both together in $22.7 \%$ of patients. The mean GIF score for the first 3 days in the ICU was identified as an independent risk factor for mortality (odds ratio $=3.02,95 \%$ confidence interval $=1.63$ to 5.59; P $<0.001$ ). The GIF score integrated into the SOFA score allowed better prediction of ICU mortality than did the SOFA score alone, and was an independent predictor of mortality (odds ratio $=1.49,95 \%$ confidence interval $=1.28$ to $1.74 ; \mathrm{P}<0.001)$. The development of gastrointestinal failure $(\mathrm{FI}$ plus IAH) was associated with significantly higher ICU and 90day mortality.

Conclusion The GIF score is useful for classifying information on the gastrointestinal system. The mean GIF score during the first 3 days in the ICU had high prognostic value for ICU mortality. Development of gastrointestinal failure is associated with significantly impaired outcome.

\section{Introduction}

Gastrointestinal problems occur frequently and are associated with adverse outcomes in critically ill patients [1-4]. Despite this, there is no consensual means for obtaining a precise assessment of gastrointestinal function. Furthermore, gastrointestinal function is not included in any of the scoring systems widely used to assess organ failure in critical illness. That the importance of gastrointestinal failure in critically ill patients is underestimated is clear from its lack of clear definition.
Numerous, mostly primary diagnosis-based definitions have been used by various investigators, making comparative interpretation of studies of gastrointestinal function rather difficult, if not impossible [5].

More than 10 years ago the summary of a roundtable conference in gut dysfunction in critical illness [6] concluded that intestinal function is an important determinant of outcome in critically ill patients; that there is no objective, clinically relevant

$\overline{\mathrm{ACS}}=$ abdominal compartment syndrome; APACHE = Acute Physiology and Chronic Health Evaluation; Cl = confidence interval; FI = food intolerance; GIF = Gastrointestinal Failure (score); IAH = intra-abdominal hypertension; IAP = intra-abdominal pressure; ICU = intensive care unit; OR = odds ratio. 
definition of intestinal dysfunction in critical illness; and that any definition developed in the future should grade the severity of the dysfunction. A scoring system for gastrointestinal dysfunction is thus warranted, and the continuing lack of a systamtic approach is limiting studies conducted to assess epidemiology, time course, risk factors and treatment.

Different gastrointestinal complications (decreased bowel sounds, delayed gastric emptying, and diarrhoea) may occur in up to $50 \%$ of mechanically ventilated patients $[1,7]$. Intolerance to gastric feeding due to delayed gastric emptying occurs in approximately half of critically ill patients [2,7-10] and has an adverse impact on intensive care unit (ICU) mortality and length of stay $[2,7,9]$.

Monitoring of intra-abdominal pressure (IAP) is gaining greater popularity in everyday clinical practice. It is easily performed and yield a reliable value that may be interpreted. Several studies have demonstrated the impact that intra-abdominal hypertension (IAH) has on mortality [11-13]. However, IAP has not been proven to represent an adequate measure of gastrointestinal function, and there is some evidence suggesting that not all patients with $\mathrm{IAH}$ have gastrointestinal problems and vice versa [14].

Based on the findings described above, we hypothesized that a combination of IAP and gastrointestinal symptoms might be a good basis for evaluating gastrointestinal dysfunction in critically ill patients. With the goal of developing a scoring system for gastrointestinal failure, we combined gastrointestinal symptoms and IAH into a 5-grade scale - the Gastrointestinal Failure (GIF) score. Similar to other organ failure scores, the instrument requires validation within the setting of an assessment of mortality among patients with different GIF scores.

The aim of the present study was to test the accuracy of the GIF score. We examined GIF as a part of multiple organ failure in a population of mixed ICU patients by evaluating the prognostic value of the GIF score both alone and in combination with the Sequential Organ Failure Assessment (SOFA) score.
We also aimed to describe the incidence and outcome of gastrointestinal failure according to GIF score.

\section{Materials and methods}

All mechanically ventilated patients subsequently admitted to the mixed surgical-medical ICU of Tartu University Hospital from September 2006 to September 2007 were screened for inclusion in the present prospective study. Patients treated for at least 24 hours were included in further analyses. On admission, the following parameters were recorded: age, sex, body mass index, readmission rate, diabetes, Acute Physiology and Chronic Health Evaluation (APACHE II) score [15], surgical profile, and whether laparatomy was performed (immediately before ICU admission or during the first 24 hours). The SOFA score [16], mean arterial pressure, central venous pressure, peak inspiratory pressure, positive end-expiratory pressure, IAP, lactate, fluid gain, use of vasopressor/inotrope and sedation were recorded on daily basis. Gastrointestinal function of the patients was assessed daily using the GIF score, described in Table 1.

Enteral feeding was started as early as possible, but not within the first days after major abdominal surgery. Food intolerance (FI) was diagnosed when applied enteral feeding appeared to be unsuccessful and had to be discontinued because of repeated or profuse vomiting, high gastric residuals, ileus, severe diarrhoea, abdominal pain, or distension. FI was not registered when the patient was electively not fed during the first 3 days after laparatomy. Gastric residual volume was considered to be high when it exceeded the volume previously given enterally.

IAP was measured via the bladder, with patients in the supine position, using the closed loop system repeated measurements technique [17]. The IAP was measured at least twice a day when normal values were recorded, and at least four times a day if IAP was found to be elevated above $12 \mathrm{mmHg}$. Mean and maximum values of IAP were documented daily. Mean IAP was used to calculate daily GIF score. IAH was defined as an IAP that was persistently $12 \mathrm{mmHg}$ or greater [18]. Abdominal compartment syndrome was defined as an IAP that was per-

Table 1

\begin{tabular}{ll}
\hline GIF score & \\
\hline Points & Clinical symptomatology \\
\hline 0 & Normal gastrointestinal function \\
1 & $\begin{array}{l}\text { Enteral feeding }<50 \% \text { of calculated needs or no feeding } 3 \text { days after abdominal surgery } \\
2\end{array}$ \\
$\begin{array}{l}\text { Food intolerance (enteral feeding not applicable due to high gastric aspirate volume, vomiting, bowel distension, or severe diarrhoea) } \\
\text { or IAH }\end{array}$ & $\begin{array}{l}\text { Food intolerance and IAH } \\
4\end{array}$ \\
\hline
\end{tabular}

GIF, Gastrointestinal Failure; IAH, intra-abdominal hypertension. 
sistently above $20 \mathrm{mmHg}$, along with onset of a new organ failure. Gastrointestinal failure was considered to be present when IAH and FI occurred simultaneously.

ICU, 28-day and 90-day mortality, and durations of ICU stay and mechanical ventilation were primary outcome parameters. The SOFA+GIF score was calculated each day by summarizing the SOFA score and the GIF score of the respective day in each patient.

The Ethics Committee of the University of Tartu approved the study. Written informed consent was not considered necessary for the study, because it was observational in nature. No special interventions were applied. All of the data were rendered anonymous before analysis, and no harm resulted from the study that could be weighed against benefit.

\section{Statistical analysis}

Statistical Package for the Social Sciences (version 15.0; SPSS Inc., Chicago, IL, USA) software was used for statistical analysis. $t$-test for continuous variables and $\mathrm{c}^{2}$ test for categorical variables were used for comparisons of two groups. Mean scores during the first 3 days were calculated as a mean of individual values for 3 days for every patient. Univariate analyses of admission parameters were applied to identify risk factors for ICU mortality. Parameters with $P<0.2$ were thereafter entered into the multiple logistic regression model to identify independent risk factors. The means of the variables for the first 3 days were thereafter added to the admission parameters in multiple regression analysis. The first day values of the parameters, included in the scores, were removed from this analysis to exclude coupling. Receiver operating characteristic curves were used to determine the likelihood ratios for the abilities of the GIF score, SOFA score and SOFA+GIF score to predict ICU mortality. Kaplan-Meier curves and log-rank tests were used to compare survival between patients with and those without gastrointestinal failure. Data are presented as mean (standard deviation), if not stated otherwise. $P<0.05$ was considered statstically significant.

\section{Results}

A total of 373 patients were treated in the general ICU of Tartu University Hospital during the study period; 264 patients were receiving mechanical ventilation at admission and stayed in the ICU for at least 24 hours, and were therefore included in further analysis.

Of these patients, $93.9 \%$ were admitted on an emergent basis. The case-mix does not include cardiac surgical or neurosurgical patients. Most of the surgical patients were admitted because of respiratory failure $(43 \%)$ or shock $(29 \%)$. Among medical patients, the main causes for admission were coma (30\%), shock (21\%), postresuscitation state (20\%) and respiratory failure (12\%). Admission parameters and outcome data for the included patients are presented in Table 2.

\section{Incidence of food intolerance and intra-abdominal hypertension}

FI was observed in 154 patients (58.3\%), and it developed predominantly during the first 3 days of admission (144/154 [93.5\%]). Seventy-two patients (27.3\%) developed IAH, and five of them (6.9\% of IAH patients) suffered from ACS. Of IAH patients, 63 out of 72 (87.5\%) developed the syndrome during their first 3 days in the ICU.

Gastrointestinal failure ( $\mathrm{FI}$ plus $\mathrm{IAH}$ ) developed in 60 patients (22.7\%); in 36 of them (13.6\% of the study population) it was already present on the day of admission (Table 2).

\section{Patient management}

Metoclopramide was routinely used as a prokinetic drug in case of feeding problems. All patients suffering from IAH received at least one of the noninvasive techniques suggested to decrease IAP [19]: paracentesis, continuous venovenous haemodiafiltration, laxatives, nasogastric aspiration, sedation, negative fluid balance, relaxants, rectal gas tube, or enema. Laparatomy was performed in three patients with ACS during the first 2 days; one of them (a trauma patient) survived, and two patients (pancreatitis as the primary disease) died. Both patients with ACS who did not undergo laparatomy died.

In $92 \%$ of patients, sedation was started upon admission or previously instituted sedation was continued in the ICU; $76 \%$ of patients received opiods. Enteral feeding was successfully applied in only $18.6 \%$ of patients on their admission day; $47 \%$ of patients did not tolerate enteral feeding; and feeding was not started in $34.4 \%$ of patients on admission.

\section{GIF score}

The GIF score was documented overall in 2,348 patient-days. GIF score 0 was observed in 52.0\%, 1 in $12.2 \%, 2$ in $27.8 \%$, 3 in $7.7 \%$, and 4 in $0.3 \%$ of days. Jejunal feeding was applied in $11 \%$ of all patient-days, but very rarely (1\%) during the first 3 days. Of the patients who had a GIF score of 1 for 1 day, $27.6 \%$ developed higher GIF scores later. In patients who had a GIF score of 1 for 2 or more subsequent days, progression of the syndrome was more common; $72.3 \%$ of them subsequently developed higher GIF scores during the following days. The mean \pm standard deviation GIF score during first 3 days in the ICU was $1.2 \pm 0.9$ points, being significantly different between survivors and nonsurvivors $(1.1 \pm 0.8$ versus 2.0 \pm 1.0 , respectively; $P<0.001)$. The mean of the maximum GIF score was $1.6 \pm 1.0$ in survivors versus $2.3 \pm 1.1$ in nonsurvivors $(P<0.001)$.

\section{Outcome}

ICU mortality in the study population was $14.8 \%$. At 28 and 90 days the mortality rates were $20.5 \%$ and $28.4 \%$, respectively. The lengths of ICU stay and mechanical ventilation, and ICU and 90-day mortality rates were significantly different between 
Critical Care Vol 12 No 4 Reintam et al.

Table 2

Admission and outcome parameters of study patients

\begin{tabular}{|c|c|c|c|c|c|c|c|}
\hline \multirow[t]{2}{*}{ Admission parameters } & \multirow[b]{2}{*}{ Total } & \multicolumn{3}{|c|}{$\mathrm{IAH}$} & \multicolumn{3}{|c|}{$\mathrm{Fl}$} \\
\hline & & Absent & Present & $P$ & Absent & Present & $P$ \\
\hline Number of patients (\%) & $264 \pm 100.0$ & $192 \pm 72.3$ & $72 \pm 27.3$ & & $110 \pm 41.7$ & $154 \pm 58.3$ & \\
\hline Male sex $(n[\%])$ & $166 \pm 62.9$ & $117 \pm 60.9$ & $49 \pm 68.1$ & 0.178 & $60 \pm 54.5$ & $106 \pm 68.8$ & 0.013 \\
\hline Age (years) & $53.8 \pm 20.0$ & $52.3 \pm 21.0$ & $57.8 \pm 16.8$ & 0.047 & $48.6 \pm 21.0$ & $57.5 \pm 18.5$ & $<0.001$ \\
\hline Diabetes $(n[\%])$ & $35 \pm 13.3$ & $26 \pm 13.5$ & $9 \pm 12.5$ & 0.502 & $11 \pm 10.0$ & $24 \pm 15.6$ & 0.128 \\
\hline Readmission ( $n[\%])$ & $8 \pm 3.0$ & $6 \pm 3.1$ & $2 \pm 2.8$ & 0.622 & $2 \pm 1.8$ & $6 \pm 3.9$ & 0.287 \\
\hline Sedation $(n[\%])$ & $243 \pm 92.0$ & $174 \pm 90.6$ & $69 \pm 95.8$ & 0.125 & $98 \pm 89.1$ & $145 \pm 94.2$ & 0.103 \\
\hline Vasoactive/inotrope (n [\%]) & $200 \pm 75.8$ & $136 \pm 70.8$ & $64 \pm 88.9$ & 0.001 & $67 \pm 60.9$ & $133 \pm 86.4$ & $<0.001$ \\
\hline Surgical profile ( $n[\%])$ & $175 \pm 66.3$ & $122 \pm 63.5$ & $53 \pm 73.7$ & 0.080 & $67 \pm 60.9$ & $108 \pm 70.1$ & 0.077 \\
\hline Laparatomy ( $n[\%])$ & $60 \pm 23.3$ & $21 \pm 15.6$ & $31 \pm 43.7$ & $<0.001$ & $9 \pm 8.4$ & $51 \pm 34.0$ & $<0.001$ \\
\hline Enteral feeding ( $n[\%])$ & $49 \pm 18.6$ & $42 \pm 21.9$ & $7 \pm 9.7$ & 0.015 & $25 \pm 22.7$ & $24 \pm 15.6$ & 0.095 \\
\hline APACHE II score (points) & $14.2 \pm 7.7$ & $13.5 \pm 7.6$ & $16.0 \pm 7.7$ & 0.016 & $11.8 \pm 7.3$ & $15.8 \pm 7.6$ & $<0.001$ \\
\hline BMI $\left(\mathrm{kg} / \mathrm{m}^{2}\right)$ & $27.6 \pm 13.1$ & $25.4 \pm 4.6$ & $33.1 \pm 22.2$ & $<0.001$ & $25.8 \pm 5.6$ & $28.9 \pm 16.4$ & 0.080 \\
\hline SOFA score (points) & $7.0 \pm 4.2$ & $6.3 \pm 4.2$ & $8.9 \pm 3.6$ & $<0.001$ & $5.3 \pm 4.2$ & $8.2 \pm 3.8$ & $<0.001$ \\
\hline Fluid gain in first 24 hours (I) & $2.4 \pm 3.6$ & $2.0 \pm 2.4$ & $3.6 \pm 5.5$ & 0.001 & $1.7 \pm 2.4$ & $2.9 \pm 4.2$ & 0.007 \\
\hline MAP $(\mathrm{mmHg})$ & $81.5 \pm 15.9$ & $82.1 \pm 16.4$ & $80.5 \pm 15.0$ & 0.512 & $82.1 \pm 15.8$ & $81.3 \pm 16.1$ & 0.758 \\
\hline IAP $(\mathrm{mmHg})$ & $8.5 \pm 4.7$ & $6.5 \pm 3.8$ & $12.1 \pm 4.0$ & $<0.001$ & $6.3 \pm 4.0$ & $9.5 \pm 4.6$ & $<0.001$ \\
\hline CVP $(\mathrm{mmHg})$ & $11.6 \pm 5.7$ & $10.3 \pm 4.9$ & $14.3 \pm 6.3$ & $<0.001$ & $10.4 \pm 5.1$ & $12.2 \pm 5.9$ & 0.031 \\
\hline $\mathrm{PIP}\left(\mathrm{cmH}_{2} \mathrm{O}\right)$ & $23.9 \pm 6.2$ & $22.6 \pm 6.0$ & $27.2 \pm 5.2$ & $<0.001$ & $22.5 \pm 6.5$ & $24.8 \pm 5.7$ & 0.006 \\
\hline PEEP $\left(\mathrm{cmH}_{2} \mathrm{O}\right)$ & $9.2 \pm 4.2$ & $8.5 \pm 4.1$ & $11.2 \pm 3.7$ & $<0.001$ & $7.8 \pm 4.1$ & $10.2 \pm 3.9$ & $<0.001$ \\
\hline Lactate $(\mathrm{mmol} / \mathrm{l})$ & $4.6 \pm 5.4$ & $4.4 \pm 4.6$ & $4.9 \pm 6.9$ & 0.519 & $4.4 \pm 4.9$ & $4.7 \pm 5.7$ & 0.741 \\
\hline $\mathrm{FI}(n[\%])$ & $124 \pm 47.0$ & $69 \pm 35.9$ & $55 \pm 76.4$ & $<0.001$ & - & - & \\
\hline $\mathrm{IAH}(n[\%])$ & $42 \pm 15.9$ & - & - & & $3 \pm 2.7$ & $39 \pm 25.3$ & $<0.001$ \\
\hline Gastrointestinal failure $(\mathrm{FI}+\mathrm{IAH} ; n[\%])$ & $36 \pm 13.6$ & - & $36 \pm 50.0$ & & - & $36 \pm 23.4$ & \\
\hline \multicolumn{8}{|l|}{ Outcome parameters } \\
\hline MV (days) & $7.4 \pm 11.9$ & $4.3 \pm 6.1$ & $15.5 \pm 18.0$ & $<0.001$ & $3.6 \pm 5.9$ & $10.0 \pm 14.1$ & $<0.001$ \\
\hline ICU (days) & $8.8 \pm 12.8$ & $5.6 \pm 7.3$ & $17.5 \pm 19.0$ & $<0.001$ & $4.8 \pm 6.9$ & $11.7 \pm 15.1$ & $<0.001$ \\
\hline ICU mortality ( $n[\%])$ & $39 \pm 14.8$ & $21 \pm 10.9$ & $18 \pm 25.0$ & 0.005 & $7 \pm 6.4$ & $32 \pm 20.8$ & 0.001 \\
\hline 28-day mortality $(n[\%])$ & $54 \pm 20.5 \%$ & $36 \pm 18.8 \%$ & $18 \pm 25.0 \%$ & 0.171 & $13 \pm 11.8 \%$ & $41 \pm 26.6 \%$ & $<0.001$ \\
\hline 90-day mortality ( $n[\%])$ & $75 \pm 28.4 \%$ & $48 \pm 25.0 \%$ & $27 \pm 37.5 \%$ & 0.033 & $17 \pm 15.5 \%$ & $58 \pm 37.7 \%$ & 0.002 \\
\hline
\end{tabular}

The values are presented as mean \pm standard deviation. APACHE, Acute Physiology and Chronic Health Evaluation; BMl, body mass index; CVP, central venous pressure; FI, food intolerance; IAH, intra-abdominal hypertension; IAP, intra-abdominal pressure; ICU, intensive care unit; MAP, mean arterial pressure; MV, mechanical ventilation; PEEP, positive end-expiratory pressure; PIP, peak inspiratory pressure; SOFA, Sequential Organ Failure Assessment.

$\mathrm{IAH}$ and non-IAH patients, as well as between $\mathrm{FI}$ and non- $\mathrm{FI}$ patients (Table 2).

A high mean GIF score during the first 3 days of the ICU stay was associated with a high rate of mortality (Figure 1). The patients with gastrointestinal failure (simultaneous occurrence of $\mathrm{IAH}$ and $\mathrm{FI}$ ) suffered from an ICU mortality of $28.1 \%$, as compared with $10.8 \%$ in patients without this syndrome $(P=$ $0.001)$. The mortality was also higher after 90 days $(40.0 \%$ versus $25.0 \% ; P=0.019)$, but not after 28 days $(28.3 \%$ versus $18.1 \% ; P=0.065)$. 
Figure 1

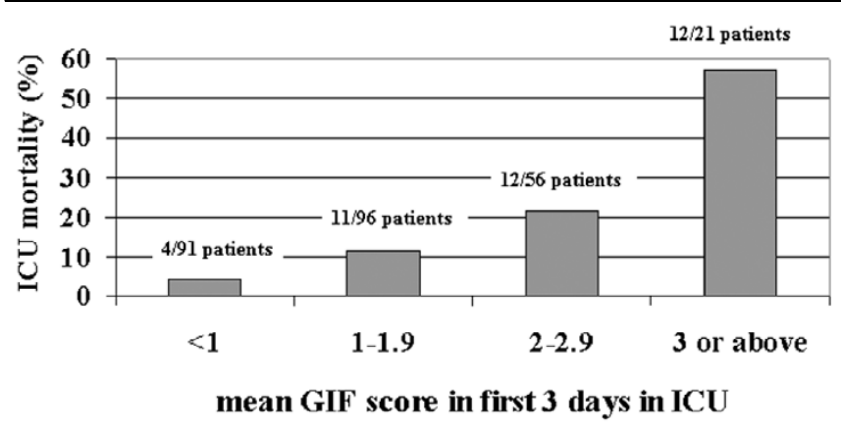

ICU mortality of patients according to their mean GIF score. ICU, intensive care unit; GIF, Gastrointestinal Failure (score).

\section{Prediction of outcome}

Admission parameters and prediction of ICU mortality

In multiple regression analysis, only two admission parameters (SOFA and fluid balance during the first 24 hours) were identified as independent predictors of ICU mortality in the study population.

Means of the 3 three days in combination with admission parameters

As expected, the mean SOFA score for the first 3 days was a better predictor than its value on the first day (odds ratio [OR] $=1.40,95 \%$ confidence interval $[\mathrm{Cl}]=1.18$ to $1.64[P<$ $0.001]$ versus $\mathrm{OR}=1.36,95 \% \mathrm{Cl}=1.02$ to $1.82[P=$ $0.037])$. The cumulative fluid balance, mean IAP and development of $\mathrm{Fl}$ (yes/no) during the first 3 days had significant impacts in univariate analyses, but they were not identified as independent risk factors for mortality.

The mean GIF score during the first 3 days (used instead of the mean IAP and development of FI) was identified as an independent risk factor for ICU mortality $(\mathrm{OR}=3.02,95 \% \mathrm{Cl}$ $=1.63$ to $5.59 ; P<0.001)$. The mean SOFA+GIF score for the first 3 days demonstrated slightly better prediction of ICU mortality than did the SOFA score alone $(\mathrm{OR}=1.49,95 \% \mathrm{Cl}$ $=1.28$ to $1.74 ; P<0.001)$.

\section{Combination of GIF and SOFA scores}

The combination of mean SOFA and GIF score during the first 3 days exhibited the greatest area under the curve (0.895), being superior to those for the mean SOFA score $(0.840)$ and the mean GIF score (0.753) alone (Figure 2). In the regression analysis for prediction of ICU mortality (Table 3), the GIF score for first 3 days had the second highest OR $(2.20,96 \% \mathrm{Cl}=$ $1.28-3.78 ; P=0.004)$, after the cardiovascular SOFA subscore $(\mathrm{OR}=5.91, \mathrm{Cl}=2.83$ to $12.33 ; P<0.001)$.

Gastrointestinal failure (intra-abdominal hypertension plus food intolerance) and outcome

The 90-day cumulative survival of patients with gastrointestinal failure was significantly impaired in comparison with patients
Figure 2

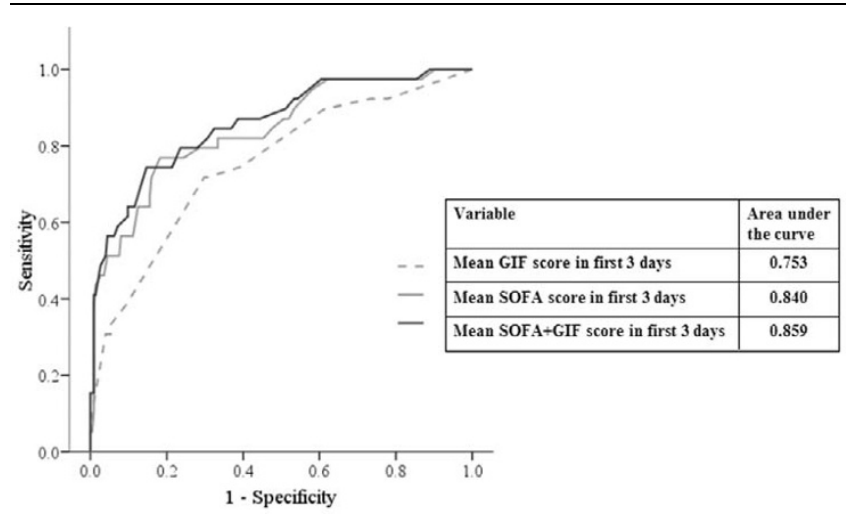

ROC curves with different scores in prediction of ICU mortality. ICU, intensive care unit; ROC, receiver operating characteristic.

without gastrointestinal failure (log-rank test $=4.45 ; P=$ $0.035)$. There was no significant difference in 28-day survival, as shown in Figure 3 . Ten patients with gastrointestinal failure (15.6\%) were still in the ICU on day 28, and three of them died in the ICU, whereas none of the patients without GIF died in the ICU after day 28.

\section{Discussion}

This single-centre pilot study demonstrates the usefulness of the GIF score - a combined assessment of FI and IAP - for dynamic assessment of gastrointestinal function in critically ill patients. Combining FI with IAP values appeared to be a better predictor of outcome than either of these parameters alone. The mean GIF score of the first 3 days is an independent risk factor for ICU mortality. Furthermore, the score may add predictive power to the SOFA score in outcome prediction.

Gastrointestinal function was demonstrated to influence the ICU outcome in previous studies. However, the absence of a scaled system for assessing gastrointestinal function has been a major limiting factor in these studies. The role of the

\section{Table 3}

SOFA subscores and GIF score in regression analysis for prediction of ICU mortality

\begin{tabular}{llll}
\hline Score/subscore & $P$ & OR & $95 \% \mathrm{Cl}$ \\
\hline Cardiovascular SOFA & $<0.001$ & 5.91 & $2.83-12.33$ \\
GIF score & 0.004 & 2.20 & $1.28-3.78$ \\
Hepatic SOFA & 0.024 & 1.75 & $1.075-2.86$ \\
Renal SOFA & 0.087 & 1.39 & $0.95-2.04$ \\
Central nervous system SOFA & 0.159 & 1.23 & $0.92-1.65$ \\
Haematological SOFA & 0.712 & 0.92 & $0.57-1.47$ \\
Respiratory SOFA & 0.518 & 0.84 & $0.48-1.44$
\end{tabular}

$\mathrm{Cl}$, confidence interval; GIF, Gastrointestinal Failure (score); OR, odds ratio; SOFA, Sequential Organ Failure Assessment. 
Figure 3

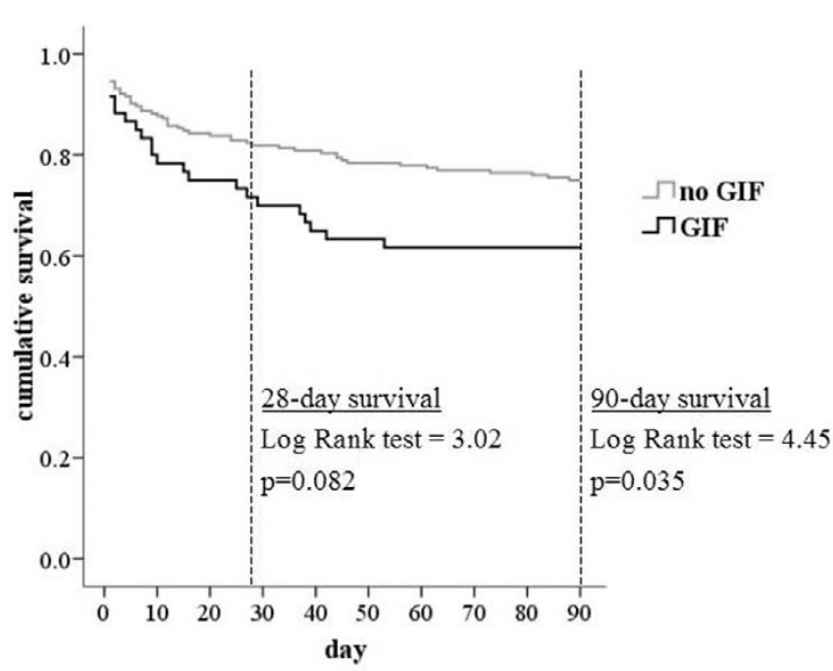

Survival: absence versus presence of gastrointestinal failure. Shown is the cumulative survival of patients without gastrointestinal failure (maximum Gastrointestinal Failure [GIF] score during intensive care unit $(I C U)$ stay $\leq 2$ ) versus patients with gastrointestinal failure (maximum GIF score during ICU stay of 3 or 4 ).

gastrointestinal tract as a motor of multiple organ failure was identified more than 2 decades ago and was more recently confirmed by Clark and Coopersmith [20]. Nevertheless, because of a lack of definition, diagnostic reliability $[21,22]$ and accurate assessment of incidence, gastrointestinal failure is not included in severity of illness scoring systems currently in use.

About half of the patients of included in the present study developed $\mathrm{FI}$ during the first 3 days after ICU admission. These patients were significantly older and more severely ill (higher APACHE and SOFA scores). They also stayed longer in ICU and exhibited greater mortality than did patients with normal gastrointestinal function. The prevalence of $\mathrm{Fl}$ was described to be within a similar range in the literature and has been shown to influence outcomes $[2,7,8]$. Mortality of food intolerant patients in the present study was lower than in previous studies, with mortality ranging between $30 \%$ to $40 \%$ $[2,7]$, but also significantly higher than in patients without $\mathrm{FI}$.

At first glance, it appears reasonable to use more specific gastrointestinal symptoms, such as bleeding, high gastric residual volumes and so on, when assessing gastrointestinal function. It should be noted, however, that there are a number of obstacles to their use in this setting: gastrointestinal bleeding occurs rarely $[22,23]$; the incidence of vomiting is influenced by nasogastric aspiration; and high gastric residual volume is not defined uniformly and exhibits only a weak correlation with gastric emptying [23]. None of these potential markers of gastrointestinal function take into account factors such as severe diarrhoea, which is often treated by reducing the rate of enteral feeding [1] and has been shown to double the likelihood of graft loss and patient death after kidney transplantation [24].

Most attempts to define gastrointestinal dysfunction described in the literature are based on diagnosis rather than function. For example, the presence of cholecystitis [25] and gastrointestinal bleeding $[22,25]$ were previously suggested to identify GIF. Such approaches do not consider a functional assessment of the gastrointestinal tract - a highly complex organ. Surprising variability also exists in definitions of $\mathrm{Fl}$. Although most authors define it based on high gastric residuals or vomiting $[2,9,23,26]$, others also include include abdominal pain or distension and diarrhoea as reasons to stop feeding and declare FI to be present [27]. Even though FI is a rather subjective variable, it is - in our opinion - the most universally used clinical characteristic of gastrointestinal failure, probably covering the entire spectrum of gastrointestinal symptoms. As has also been stated by the experts, despite obvious limitations to the definition of intolerance to enteral feeding, it provides a functional assessment with some clinical relevance [6].

$\mathrm{IAH}$ did not occur in our patients as frequently as $\mathrm{FI}$; it developed in only one-third of them. These data are in accordance with observations from Malbrain and colleagues [28], who described a similar prevalence of IAH in a mixed ICU population. Various studies conducted in selected patients groups have identified an adverse impact of IAP on ICU outcome $[13,29]$. Malbrain and coworkers [11] found the development of IAH during the ICU stay, but not IAP on admission, to be an independent risk factor for mortality [11]. However, prediction of outcome based on events occurring during the entire ICU period is of somewhat limited value. Therefore, we limited our assessment to mean values during the first 3 days. Accordingly, the GIF score, but not IAH or Fl, appeared to be an independent predictor of outcome.

Little is known about the combination of FI with IAH. Our data clearly demonstrate that patients manifesting these two signs are not fully overlapping groups; not all the patients with gastrointestinal problems have IAH and vice versa. Of the patients with IAH on admission, $76 \%$ also experienced $\mathrm{FI}$, whereas only $25 \%$ the patients with $\mathrm{FI}$ had IAH. Some of the patients who subsequently developed IAH exhibited $\mathrm{FI}$ on admission, and only a few patients who went on to develop FI exhibited IAH on admission. This, in our opinion, further supports the need to combine these two variables in the GIF score. The definite strength of IAP measurement in this setting is its objective and reproducibly measurable numeric value.

It is difficult to estimate the extent to which the route of enteral feeding influences the GIF score. However, the advantage of post-pyloric versus gastric feeding with respect to outcome is not yet established [30], and thus the current evidence does not support routine use of post-pyloric feeding in the critically 
ill [31]. The post-pyloric route is probably not the most common choice during the first few days of intensive care, even though Montejo and coworkers [3] reported a lower incidence of gastrointestinal complications in patients receiving early jejunal nutrition. It may be speculated that enteral feeding itself produces an increase in IAP in critically ill patients, but we did not observe such an association in a preliminary study [32]. Feeding and sedation strategies are expected to influence gastrointestinal function. However, if we are to evaluate the impact that different treatments have, we require a tool for evaluating gastrointestinal dysfunction.

The main limitation of the present study is that only those patients with prolonged ICU stay ( $>24$ hours) were included. Patients treated in the ICU for less than 24 hours arguably contain a mixture of the least and most severely ill patients. This pre-selection may bias the results, which might account for the low predictive power of the APACHE II score. However, in most short-staying patients the IAH and Fl are not usually the key issues of the treatment. IAH is seldom measured in patients who die within a few hours after ICU admission. Recognizing this delay in IAH monitoring, it is important to emphasize that in a few ACS patients prompt IAH measurement might be crucial to making the correct therapeutic decisions and thus maximizing survival. Parsak and coworkers [33] recently demonstrated an advantage of early surgical intervention in patients with primary ACS, and they emphasized the importance of controlling IAP during the early postoperative period. Although mortality from ACS remains high, the avoidance of this end-stage syndrome may be possible with IAP monitoring and treatment strategies recommended by the World Society of the Abdominal Compartment Syndrome [34].

The observed high predictive value of the mean SOFA score for ICU outcome is in accordance with the findings of several previous studies. The predictive power of the mean SOFA score during the first 3 days is correctly placed between the those of the mean SOFA score for the whole ICU period (OR $=3.06)$ and the SOFA score at 48 hours $(O R=1.45)$ [35]. Similar predictive value of SOFA subscores was observed in cardiac surgical patients [36]. The cardiovascular SOFA score appeared to be the most powerful, whereas the respiratory and haematological SOFA scores were the least powerful [36]. The excellent performance of the GIF score in this setting once more confirms the importance of gastrointestinal failure among other organ failures. The cumulative survival curves of patients with or without GIF further emphasize this finding. The fact that the difference in favour of patients without GIF is significant with respect to 90-day survival, but not survival at 28 days, is probably accounted for by the longer ICU stay with subsequently higher ICU mortality in GIF patients.
A limitation of this study is its single-centre design. The GIF score is probably influenced by both case-mix and treatment strategies; therefore, variation between centres may occur.

In our opinion, the major limitation of the GIF score is the subjectivity of estimation of the presence of $\mathrm{Fl}$. There is no consensual definition of $\mathrm{Fl}$, and variability in the definitions used in the literature is considerable. Also, continuity of the variables in the GIF score could be improved. The score is not exactly a continuum of alterations, as suggested for an organ failure score by Ferreira and coworkers [35]. However, it does fulfill the other criterion established by that author; specifically, it is based on easily accessible variables [35]. As the mean score of the first 3 days is not very helpful in everyday ICU practice, we propose a possible interpretation of the daily GIF score by reference to the RIFLE classification [37] as follows: Risk, GIF score 1 for at least 2 days; Injury, GIF score 2; Failure, GIF score 3; and End-stage, GIF score 4.

\section{Conclusion}

The mean GIF score during the first 3 days on the ICU exhibited high prognostic value in terms of predicting ICU mortality. The GIF score is useful for categorizing information on the gastrointestinal system. Development of gastrointestinal failure during the ICU stay is associated with significantly higher ICU and 90-day mortality. Further multicentre studies should confirm whether the GIF score could be adopted as a useful subscore for gastrointestinal tract assessment in the SOFA score.

\section{Key messages}

- Gastrointestinal failure is associated with adverse outcomes.

- Gastrointestinal failure is an important part of multiple organ failure.

- $\quad \mathrm{FI}$ and IAH may be used together to evaluate the gastrointestinal system in critically ill patients.

- The GIF score is useful for categorizing information on the gastrointestinal system.

- The GIF score has high predictive value for ICU mortality.

\section{Competing interests}

The authors declare that they have no competing interests.

\section{Authors' contributions}

AR participated in designing the study and statistical analysis, and drafted the manuscript. PP and RK conducted data collection and participated in the statistical analysis. JS participated in designing the study and writing of the manuscript. HK participated in designing the study and helped to draft the manuscript. All authors read and approved the final manuscript. 


\section{Acknowledgements}

This work was supported by Estonian Science Foundation grant no. 6950. We thank Christian Heath and Martin Björck for their

contributions.

\section{References}

1. Mutlu GM, Mutlu EA, Factor P: GI complications in patients receiving mechanical ventilation. Chest 2001, 119:1222-1241.

2. Mentec H, Dupont H, Bocchetti M, Cani P, Ponche F, Bleichner G: Upper digestive intolerance during enteral nutrition in critically ill patients: frequency, risk factors, and complications. Crit Care Med 2001, 29:1955-61.

3. Montejo JC, Grau T, Acosta J, Ruiz-Santana S, Planas M, GarciaDe-Lorenzo A, Mesejo A, Cervera M, Sanchez-Alvarez C, NunezRuiz R, Lopez-Martinez J, Nutritional and Metabolic Working Group of the Spanish Society of Intensive Care and Coronary Units: Multicenter, prospective, randomized, single-blind study comparing the efficacy and gastrointestinal complications of early jejunal feeding with early gastric feeding in critically ill patients. Crit Care Med 2002, 30:796-800.

4. Reintam A, Parm P, Redlich U, Tooding LM, Starkopf J, Köhler F, Spies $\mathrm{C}$, Kern $\mathrm{H}$ : Gastrointestinal failure in intensive care: a retrospective clinical study in three different intensive care units in Germany and Estonia. BMC Gastroenterology 2006, 6:19.

5. Reintam A, Kern H, Starkopf J: Defining gastrointestinal failure. Acta Clin Belg Supp/ 2007:168-172.

6. Rombeau JL, Takala J: Summary of round table conference: gut dysfunction in critical illness. Intensive Care Med 1997, 23:476-479.

7. Montejo JC: Enteral nutrition-related gastrointestinal complications in critically ill patients: a multicenter study: the Nutritional and Metabolic Working Group of the Spanish Society of Intensive Care Medicine and Coronary Units. Crit Care Med 1999, 27:1447-1453.

8. Nguyen NQ, Ng MP, Chapman M, Fraser RJ, Holloway RH: The impact of admission diagnosis on gastric emptying in critically ill patients. Crit Care 2007, 11:R16.

9. Lam SW, Nguyen NQ, Ching K, Chapman M, Fraser RJ, Holloway $\mathrm{RH}$ : Gastric feed intolerance is not increased in critically ill patients with type II diabetes mellitus. Intensive Care Med 2007, 33:1740-1745.

10. Heyland D, Cook DJ, Winder B, Brylowski L, Van deMark H, Guyatt G: Enteral nutrition in the critically ill patient: a prospective survey. Crit Care Med 1995, 23:1055-1060.

11. Malbrain ML, Chiumello D, Pelosi $P$, Bihari D, Innes R, Ranieri VM, Del Turco M, Wilmer A, Brienza N, Malcangi V, Cohen J, Japiassu A, De eulenaer BL, Daelemans R, Jacquet L, Laterre PF, Frank G, de Souza P, Cesana B, Gattinoni L: Incidence and prognosis of intraabdominal hypertension in a mixed population of critically ill patients: a multiple-center epidemiological study. Crit Care Med 2005, 33:315-322.

12. Reintam A, Parm P, Kitus R, Kern H, Starkopf J: Primary and secondary intra-abdominal hypertension: different incidence, time-course and impact on ICU outcome [abstract]. Acta Clin Belg Supp/ 2007:248.

13. Rosas JM, Soto SN, Aracil JS, Cladera PR, Borlan RH, Sanchez AV, Ros FB, Posa LG: Intra-abdominal pressure as a marker of severity in acute pancreatitis. Surgery 2007, 141:173-178.

14. Reintam A, Parm P, Kern H, Starkopf J: Intra-abdominal hypertension and gastrointestinal symptoms in prediction of ICU outcome [abstract]. Intensive Care Med 2006, 32:S286.

15. Knaus WA, Draper EA, Wagner DP, Zimmerman JE: APACHE II: a severity of disease classification system. Crit Care Med 1985, 13:818-829.

16. Vincent JL, Moreno R, Takala J, Willatts S, De Mendonca A, Bruining $H$, Reinhart CK, Suter PM, Thijs LG: The SOFA (Sepsisrelated Organ Failure Assessment) score to describe organ dysfunction/failure. Intensive Care Med 1996, 22:707-710.

17. Cheatham ML, Safcsak K: Intraabdominal pressure a revised method for measurement. J Am Coll Surg 1998, 186:594-595.

18. Malbrain ML, Cheatham ML, Kirkpatrick A, Sugrue M, Parr M, De Waele J, Balogh Z, Leppaniemi A, Olvera C, Ivatury R, D'Amours $\mathrm{S}$, Wendon J, Hillman $\mathrm{K}$, Johansson $\mathrm{K}$, Kolkman $\mathrm{K}$, Wilmer $\mathrm{A}$ : Results from the International Conference of Experts on Intra- abdominal Hypertension and Abdominal Compartment Syndrome. I. Definitions. Intensive Care Med 2006, 32:1722-1732.

19. De laet I, Malbrain ML: ICU management of the patient with intra-abdominal hypertension: what to do, when and to whom? Acta Clin Belg Supp/ 2007:190-199.

20. Clark JA, Coopersmith CM: Intestinal cross-talk: a new paradigm for understanding the gut as the motor of critical illness. Shock 2007, 28:384-393.

21. Lefering R, Goris RJA, van Nieuwenhoven EJ, Neugebauer E: Revision of the multiple organ failure score. Langenbeck's Arch Surg 2002, 387:14-20.

22. Mayr VD, Duenser MW, Greil V, Jochberger S, Luckner G, Ulmer $\mathrm{H}$, Friesenecker BE, Takala J, Hasibeder WR: Causes of death and determinants of outcome in critically ill patients. Crit Care 2006, 10:R154.

23. Deane A, Chapman MJ, Fraser RJ, Bryant LK, Burgstad C, Nguyen $\mathrm{NQ}$ : Mechanisms underlying feed intolerance in the critically ill: implications for treatment. World J Gastroenterol 2007, 13:3909-3917.

24. Bunnapradist $S$, Neri L, Wong W, Lentine KL, Burroughs TE, Pinsky BW, Takemoto SK, Schnitzler MA: Incidence and risk factors for diarrhea following kidney transplantation and association with graft loss and mortality. Am J Kidney Dis 2008, 51:478-486.

25. Goris RJA, te Bockhorst TPA, Nuytinck JKS, Gimbrere JSF: Multiple organ failure. Arch Surg 1985, 120:1109-1110.

26. Elpern EH, Stutz L, Peterson S, Gurka DP, Skipper A: Outcomes associated with enteral tube feedings in a medical intensive care unit. Am J Crit Care 2004, 13:221-22.

27. O'Leary-Kelley CM, Puntillo KA, Barr J, Stotts N, Douglas MK: Nutritional adequacy in patients receiving mechanical ventilation who are fed enterally. Am J Crit Care 2005, 14:222-231.

28. Malbrain ML, Chiumello D, Pelosi P, Wilmer A, Brienza N, Malcangi V, Bihari D, Innes R, Cohen J, Singer P, Japiassu A, Kurtop E, De Keulenaer BL, Daelemans R, Del Turco M, Cosimini P, Ranieri M, Jaquet L, Laterre PF, Gattinoni L: Prevalence of intra-abdominal hypertension in critically ill patients: a multicentre epidemiological study. Intensive Care Med 2004, 30:822-829.

29. Djavani K, Wanhainen A, Björck M: Intra-abdominal hypertension and abdominal compartment syndrome following surgery for ruptured abdominal aortic aneurysm. Eur J Vasc Endovasc Surg 2006, 31:581-584.

30. Ukleja A, Sanchez-Fermin M: Gastric versus post-pyloric feeding: relationship to tolerance, pneumonia risk, and successful delivery of enteral nutrition. Curr Gastroenterol Rep 2007, 9:309-316.

31. Drover JW: Gastric versus postpyloric feeding. Gastrointest Endosc Clin N Am 2007, 17:765-75.

32. Tamme K, Reintam A, Kitus R, Parm P, Starkopf J: Early enteral feeding does not increase intra-abdominal pressure in patients with secondary intra-abdominal hypertension [abstract]. Int Care Med 2007, 33(suppl 2):A0413.

33. Parsak CK, Seydaoglu G, Sakman G, Acarturk TO, Karakoc E, Hanta I, Alparslan AH, Satar S: Abdominal compartment syndrome: current problems and new strategies. World J Surg 2008, 32:13-19.

34. Cheatham ML, Malbrain ML, Kirkpatrick A, Sugrue M, Parr M, De Waele J, Balogh Z, Leppäniemi A, Olvera C, Ivatury R, D'Amors S, Wendon J, Hillman K, Wilmer A: Results from the International Conference of Experts on Intra-abdominal Hypertension and Abdominal Compartment Syndrome. Intensive Care Med 2007, 33:951-962

35. Ferreira FL, Bota DP, Bross A, Melot C, Vincent JL: Serial evaluation of the SOFA score to predict outcome in critically ill patients. JAMA 2001, 286:1754-1758.

36. Ceriani R, Mazzoni M, Bortone F, Gandini S, Solinas C, Susini G, Parodi O: Application of the Sequential Organ Failure Assessment Score to cardiac surgical patients. Chest 2003, 123:1229-1239.

37. Abosaif NY, Tolba YA, Heap M, Russel J, El Nahas AM: The outcome of acute renal failure in the intensive care unit according to RIFLE: model application, sensitivity and predictability. $A m$ J Kidney Dis 2005, 46:1038-1048. 